\title{
Dilated cardiomyopathy-linked heat shock
family D member 1 mutations cause up- regulation of reactive oxygen species and autophagy through mitochondrial dysfunction
}

\author{
Hirokazu Enomoto ${ }^{1}$, Nishant Mittal ${ }^{1}$, Takayuki Inomata ${ }^{2}$, Takuro Arimura ${ }^{3}$, \\ Tohru Izumi $^{2}$, Akinori Kimura ${ }^{3}$, Keiichi Fukuda ${ }^{1}$, and Shinji Makino ${ }^{1,4 *}$
}

${ }^{1}$ Department of Cardiology, Keio University School of Medicine, Tokyo 160-8582, Japan; ${ }^{2}$ Department of Cardiovascular Medicine, Kitasato University School of Medicine, Kanagawa 252-0374, Japan; ${ }^{3}$ Department of Molecular Pathogenesis, Tokyo Medical and Dental University, Tokyo 113-8510, Japan; and ${ }^{4}$ Health Center, Keio University, Tokyo 160-8582, Japan

Received 19 November 2018; revised 1 July 2019; editorial decision 23 April 2020; accepted 4 June 2020

Time for primary review: 23 days

Aims

During heart failure, the levels of circulatory heat shock protein family D member 1 (HSP60) increase. However, its underlying mechanism is still unknown. The apical domain of heat shock protein family D member 1 (HSPD1) is conserved throughout evolution. We found a point mutation in HSPD1 in a familial dilated cardiomyopathy (DCM) patient. A similar point mutation in HSPD1 in the zebrafish mutant, $n b$, led to loss of its regenerative capacity and development of pericardial oedema under heat stress condition. In this study, we aimed to determine the direct involvement of HSPD1 in the development of DCM.

Methods By Sanger method, we found a point mutation (Thr320Ala) in the apical domain of HSPD1, in one familial DCM paand results tient, which was four amino acids away from the point mutation (Val324Glu) in the $n b l$ mutant zebrafish. The $n b l$ mutants showed atrio-ventricular block and sudden death at 8-month post-fertilization. Histological and microscopic analysis of the $\mathrm{nb}$ mutant hearts showed decreased ventricular wall thickness, elevated level of reactive oxygen species (ROS), increased fibrosis, mitochondrial damage, and increased autophagosomes. mRNA and protein expression of autophagy-related genes significantly increased in nbl mutants. We established HEK293 stable cell lines of wild-type, nbl-type, and DCM-type HSPD1, with tetracycline-dependent expression. Compared to wild-type, both nbl- and DCM-type cells showed decreased cell growth, increased expression of ROS and autophagy-related genes, inhibition of the activity of mitochondrial electron transport chain complexes III and IV, and decreased mitochondrial fission and fusion.

Conclusion Mutations in HSPD1 caused mitochondrial dysfunction and induced mitophagy. Mitochondrial dysfunction caused increased ROS and cardiac atrophy. 


\section{Graphical Abstract}

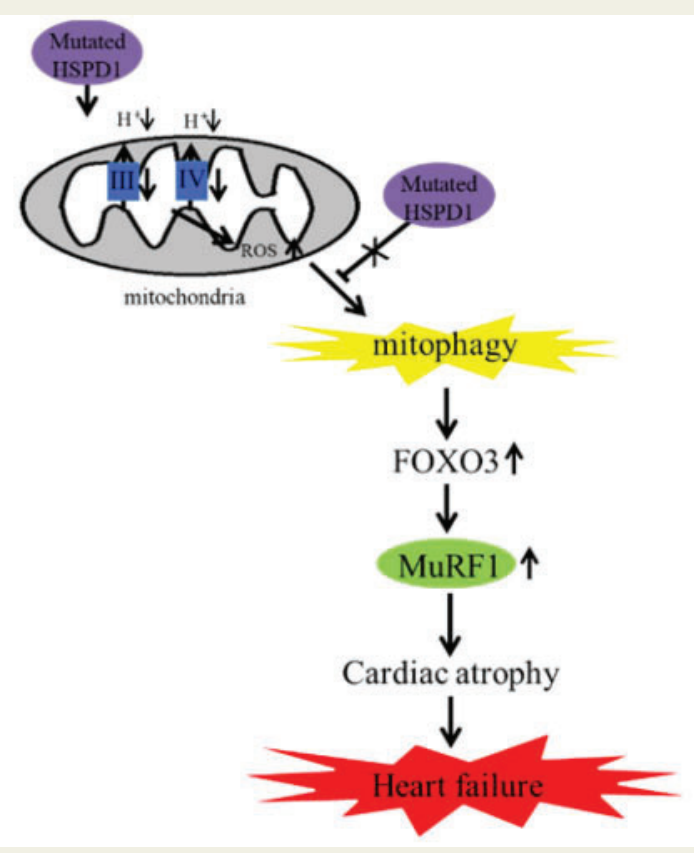

Keywords

HSPD1 • Dilated cardiomyopathy • Mitochondria • Mitophagy • Zebrafish

\section{Introduction}

Dilated cardiomyopathy (DCM) is the most frequent cause for heart transplantation. The main characteristics of DCM are heart chamber dilation and impaired systolic function. Histological examination of cardiac tissue from DCM patients usually reveals degeneration of myocytes, fibrotic remodelling, and a decrease in myofilament content. ${ }^{1}$ Heart failure is known to be related to various aetiological genetic abnormalities, mitochondrial dysfunction, excessive alcohol consumption, metabolic disorders, viral infections, and oxidative stress. ${ }^{2,3}$ Most of the genetic causes are mutations in sarcomeric specific genes and sarcomeric elements play key roles in the generation and transmission of contractile force. ${ }^{4,5}$ Moreover, DCM is known to cause arrhythmias, including atrioventricular (AV) block. These cardiac conduction abnormalities in conjunction with DCM have been reported to be related with a strong tendency to sudden death by genetic mutation.,

Heat shock protein family D member 1 (HSPD1, also named HSP60 for the orthologue in other species as zebrafish) is a well-characterized mitochondrial chaperone that prevents stress-induced protein damage and acts as a protection system of biological activity. ${ }^{8,9}$ The point mutation in HSPD1 (Asp29Gly) is reported to cause neurodegenerative disorders and hypomyelinating leukodystrophies. ${ }^{10,11}$ Interestingly, the level of HSPD1 and reactive oxygen species (ROS) is elevated in cardiac dysfunction. ${ }^{12}$ However, the underlying mechanism is unknown.

Autophagy is a cellular process that degrades long-lived proteins and recycled components. ${ }^{13}$ The tight regulation of autophagy in the heart is necessary, as either excessive activation or inhibition of autophagy significantly reduces the myocardial survival. ${ }^{14-16}$ Mitophagy, a mitochondria specific autophagy, functions as an early cardio-protective response, favouring adaptation to stress by removing damaged mitochondria. ${ }^{17}$ Another study has shown that mitophagy eliminates non-functional mitochondria in the pathogenesis of neurological Parkinson's disease. ${ }^{18}$ These studies implicate the important role of mitophagy to keep the long-lived cells healthy.

Zebrafish is an excellent model organism to study the cardiovascular system, because its embryos are transparent and can survive without blood flow through sufficient oxygen delivery by diffusion. We previously developed a zebrafish mutant, no blastema $(n b l)$, which has a Val324Glu missense mutation in exon 8 of HSPD1, leading to its loss of function. ${ }^{19}$ These mutants showed loss of regenerative capacity and misfolding of the lactate dehydrogenase protein.

In this study, we used nbl mutants as an embryonic model under heat stress conditions (acute disease models) and as adult models under optimal conditions (chronic disease models, see Supplementary material online, Figure S1). The $n b l$ mutants developed DCM-like conditions, along with enhanced ROS production and a higher number of autophagosomes. Furthermore, stable HEK293 cell lines with homogenous expression of the $n b l$ or DCM patient type of HSPD1 showed inhibition of mitochondrial complexes III and IV, as well as significant loss of mitochondrial fission and fusion. This study constitutes a first finding that the HSPD1 dysfunction decreases mitochondrial activity and develops heart failure.

\section{Methods}

\subsection{Genetic analysis of DCM patients}

Human studies were conducted in accordance with the ethical standards of the responsible committee on human experimentation (institutional 
CVR-2018-1173R3

A

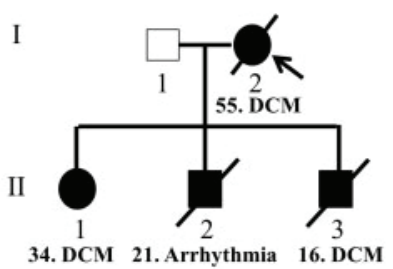

B RFLP

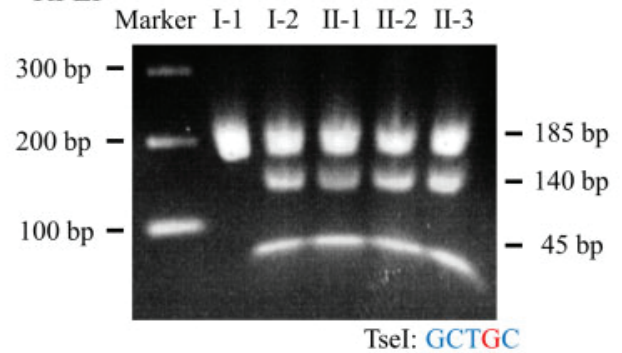

C

Control
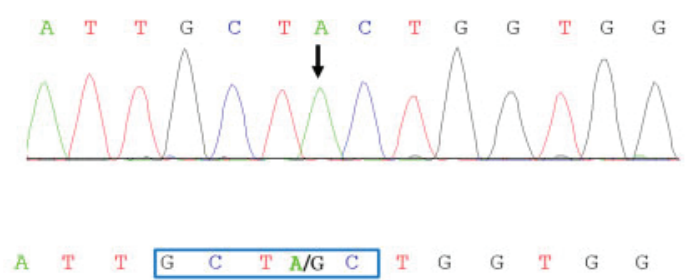

DCM patient

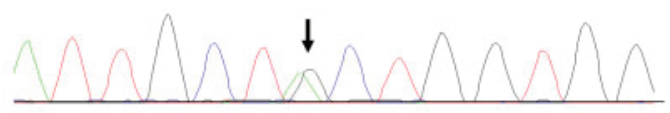

TseI: GCTGC

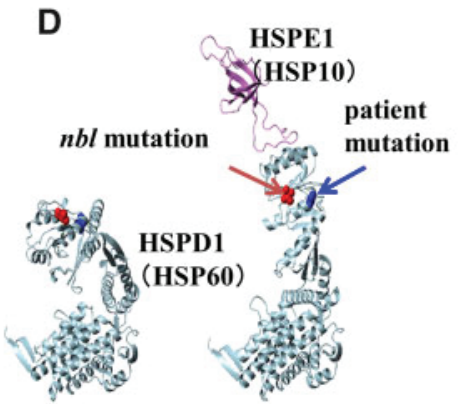

E

Homo sapiens

DCM patient

Mus musculus

Rattus norvegicus

Gallus gallus

Alligator mississippiensis

Xenopus laevis

Danio rerio

$n b l$

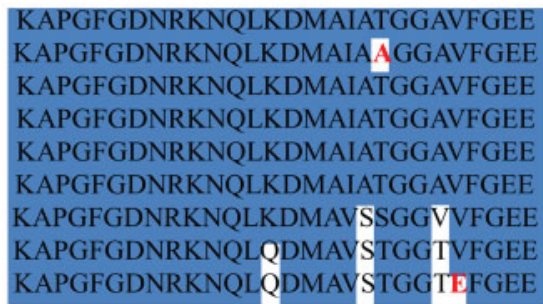

Figure I One of the DCM family had a point mutation in HSPD1. (A) We searched 96 genetically unrelated familial dilated cardiomyopathy (DCM) patients, who had no mutations in the known DCM-causing genes; one of the familial DCM patients had a point mutation in HSPD1. In that family, the proband and two of its relative died suddenly due to arrhythmia. (B) RFLP analysis for this family. (C) These familial DCM patients have a mutation in HSPD1, Thr320Ala, near the $\mathrm{nbl}$ mutation (Val324Glu) site. Red color denotes nbl point mutation. Blue color denotes DCM patient's point mutation. (D) Familial DCM and nbl-type mutation site are present on the apical domain in HSPD1. (E) Multiple species alignment of the apical domain of HSPD1 containing the nbl-and DCM-type mutations.

and national) and in accordance with the Helsinki Declaration of 1975, as revised in 2008. A total of 96 genetically unrelated Japanese patients with familial DCM were the subjects of this study. The research protocol was approved by the Ethics Review Committee of the Medical Research Institute, Tokyo Medical and Dental University, Tokyo, Japan. ${ }^{20}$ Informed consent was given prior to the inclusion of people in the study (see Supplementary material online for the detailed Methods section).

\subsection{Construction of cell lines}

HEK293 cells inducible for expression of HSPD1 wild-type (WT), HSPD1-Val324Glu (nbl), or HSPD1 Thr320Ala (DCM) were created using the Flp-In T-REx system (Invitrogen, Carlsbad, CA, USA). The cells were co-transfected with pcDNA5/FRT/TO plasmids, containing WT-, nbl- or DCM-type cDNA sequences, together with pOG44, which encodes a Flp-recombinase, according to the manufacturer's instructions. After selection with hygromycin, cells with stable integration of the pcDNA5/ FRT/TO and the vector containing the HSPD1 variants were obtained (see Supplementary material online for the detailed Cell Culture method).

\subsection{Animal procedures}

All procedures were conducted in accordance with the Animal Welfare Act and the Guide for the Care and Use of Laboratory Animals 
CVR-2018-1173R3

A

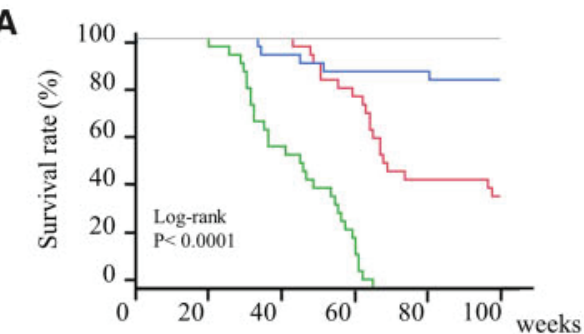

Number of survived zebrafish

$$
\begin{array}{lllllll}
30 & 30 & 28 & 26 & 26 & 25 & -\mathrm{WT} \\
30 & 30 & 30 & 24 & 14 & 12 & -\begin{array}{l}
n b l \\
\text { heterozygote }
\end{array} \\
30 & 29 & 15 & 6 & 0 & 0 & -\begin{array}{l}
n b l \\
\text { homozygote }
\end{array}
\end{array}
$$

D

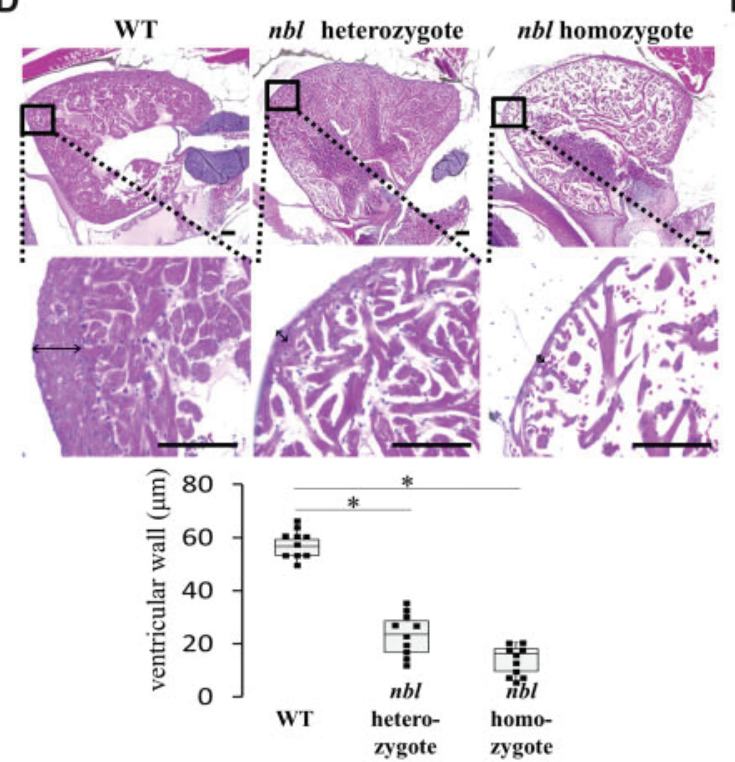

E
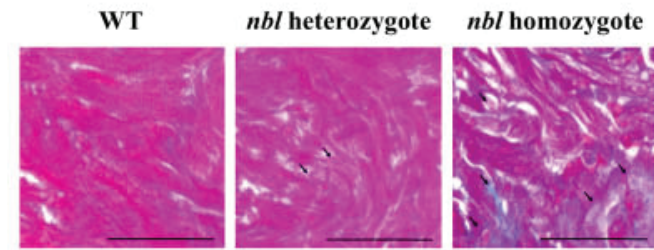

DHE
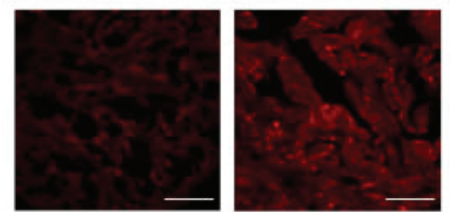

B

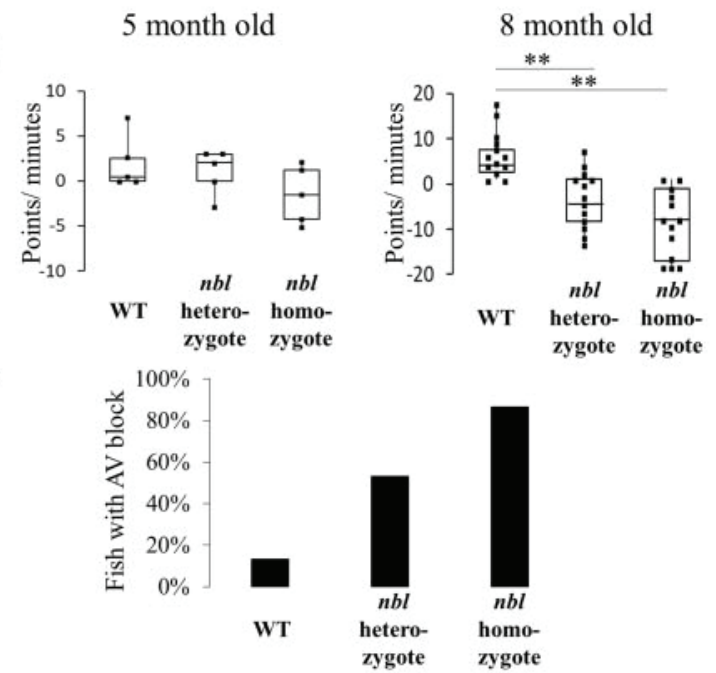

$\mathbf{F}$
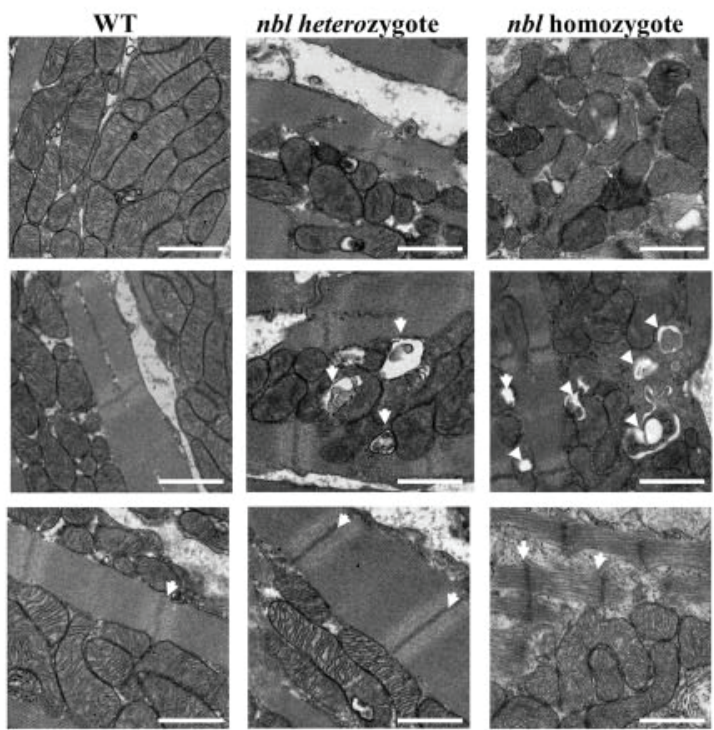

Figure 2 Phenotype of adult $n b l$ mutants grown under normal $\left(28.5^{\circ} \mathrm{C}\right)$ conditions. (A) Survival rate (Kaplan-Meier curve) of $n b l$ homozygous, heterozygous, and WT zebrafish, grown under non-heat stress condition $(n=30)$. (B) Exercise tolerance test of WT and nbl mutant 5-month post-fertilization (mpf, 
(National Institutes of Health, Bethesda, MD, USA). All experiments were approved by the local Animal Experiment Committee of CIEA of Japan (Permit number: 4790). The zebrafish were anesthetized with Ethyl 3-aminobenzoate methane sulphonate salt (Tricaine, MS-2: SigmaAldrich, St. Louis, MO, USA) followed by rapid freezingin liquid nitrogen (see Supplementary material online for the detailed Methods section).

\subsection{Chemical treatment of embryos}

For rescue assay, the $n b l$ mutant zebrafish were grown in embryonic water containing $1 \mathrm{mg} / \mathrm{ml}$ of catechin and $1 \mathrm{mM}$ of 3-methyladenine (3MA), from 12 -h post-fertilization to $120-\mathrm{h}$ post-fertilization at $34.5^{\circ}$.

\subsection{Immunohistochemistry and histology}

At the end of each experiment, the hearts and whole mount fish were collected and fixed overnight at $4{ }^{\circ} \mathrm{C}$ in $4 \%$ paraformaldehyde. The hearts were then rinsed in PBS and equilibrated in 15\% and $30 \%$ sucrose, before being embedded in Tissue-Tek OCT compound (Sakura Finetek Europe B.V., Alphen aan den Rijn, The Netherlands) and cryo-sectioned at a thickness of $6 \mu \mathrm{m}$. The immunohistochemistry procedures (haematoxylin and eosin and Azan staining) were performed as previously described $^{19,21}$ (see Supplementary material online for the detailed Methods section). The area of blue-stained collagen fibrils and redstained muscle fibre were quantified using BZ-II Analysis Software (Keyence, Japan) performed as described. ${ }^{21}$

\subsection{Transmission electron microscope}

Transmission electron microscope (TEM) was performed as described. $^{19}$

\subsection{Exercise trial}

The water flow chamber had lines at every $90^{\circ}$. We counted number of times the fish crossed these lines in $30 \mathrm{~s}$. If the fish passed through this line against the water flow, they were awarded a point. If they passed the line along with the flow, however, one point was subtracted from their score instead (see Supplementary material online, Figure S1B and C).

\subsection{Mitochondria separation and mitochondria activity}

After inducing HSPD1 expression in the cell lines, mitochondria and cytosol were separated from the various cell lines via differential sedimentation using the FOCUS SubCell (TaKaRa Bio Inc., Kusatsu, Japan). Isolated mitochondria were then resuspended in storage buffer and stored at $4^{\circ} \mathrm{C}$ to arrest their activity. About $20 \mu \mathrm{L}$ of resuspended mitochondria from the WT and mutant cell lines were immediately used as test compound for MitoCheck Complex I Activity Assay Kit, MitoCheck Complex II Activity Assay Kit, MitoCheck Complex II/III Activity Assay Kit, (Cayman Chemical, Ann Arbor, MI, USA), and MitoXpress Xtra Oxygen Consumption Assay (Luxcel Biosciences, Cork, Ireland) for mitochondria activity assay.

\subsection{HSP60/HSP10 folding activity}

After inducing HSPD1 expression in the cell lines, mitochondrial and cytosolic contents were separated via differential sedimentation using a FOCUS SubCell kit (TaKaRa Bio Inc., Kusatsu, Japan). Isolated mitochondria were then solubilized in Mitochondria Storage Buffer. HSP60/HSP10 Glow-Fold Protein Refolding kits (R\&D Systems, Inc., MA, USA) were used for HSP60/HSP10 folding activity assay, as per manufacturer's instruction with slight modification. Since we wanted to access the refolding assay with overexpressed WT and mutant type HSPD1, we used water as test compound. HSP60 solution provided in the kit was used as a positive control. We substituted the HSP60 solution provided in the kit with isolated mitochondrial solution from WT HSPD1 and mutant HSPD1 for our experiment to assess the folding activity.

\subsection{Mitochondria movie}

The three HSPD1-expressing cell lines were incubated in 1:1000 MitoGreen (TaKaRa Bio Inc., Kusatsu, Japan) medium for $30 \mathrm{~min}$. For the live imaging experiment, cells on $35 \mathrm{~mm} /$ Glass Base Dish (IWAKI, Shizuoka, JAPAN) were cultured in a small $\mathrm{CO}_{2}$ incubator (Carl Zeiss, Oberkochen, Germany) in $5 \% \mathrm{CO}_{2}$ at $37^{\circ} \mathrm{C}$, and maintained in a culture medium. Cells were scanned every $6 \mathrm{~s}$ for a duration of $2 \mathrm{~min}$ (for movies or statistical data, respectively) using an LSM 510 microscope (Carl Zeiss, Oberkochen, Germany). The values were also confirmed through naked-eye observation of the sequence of photographs that constitutes the AVI file-formatted movie.

\subsection{Statistical analysis}

Differences between groups were evaluated with the use of a two-way ANOVA to evaluate differences between individual mean values. A value of $* P<0.05, * * P<0.01$ was considered statistically significant for all comparisons. Statistical analyses for survival measurement analysis were performed with EZR (Saitama Medical Center, Jichi Medical University, Saitama, Japan), which is a graphical user interface for $R$ (The $R$ Foundation for Statistical Computing, Vienna, Austria). ${ }^{22}$ More precisely, it is a modified version of $\mathrm{R}$ commander designed to add statistical functions frequently used in biostatistics.

\section{Results}

\subsection{The point mutation in HSPD1 was found in a familial DCM patients}

We searched genetically unrelated 96 proband patients, who had no mutations in the known DCM-causing genes, with familial DCM. We identified a point mutation, Thr320Ala, in exon 8 of HSPD1 gene in one of the DCM family (Figure $1 \mathrm{~A}$ and see Supplementary material online, Figure S2). This patient, along with her three children (two sons and one daughter), had the same point mutation in HSPD1 and it was confirmed

$n=5)$ and 8-mpf $(n=13)$. Individual data points, box, and whisker plots showing the median, inter-quartile range, and maximum and minimum values. ( $C$ ) Comparison of atrio-ventricular (AV) block in $n b l$ mutants and WT, grown under non-heat stress conditions $(n=15)$. (D) Haematoxylin and eosin staining in WT, $n b l$ heterozygous, and $n b l$ homozygotes on whole mount section. $(n=10)$ Scale bar is $100 \mu \mathrm{m}$. Lower graph is thickness of ventricular wall. ( $E$ ) Azan staining and DHE staining in WT, nbl heterozygous, and homozygous mutants. Azan staining revealed fibrosis (arrowheads; fibrosis). DHE staining revealed ROS expression. We, further, quantified the rate of fibrosis and ROS activity $(n=5)$. Here, each dot indicates average of five frames per fish sample. Scale bar is $50 \mu \mathrm{m}$. (F) Electron microscopy analysis in WT, nbl heterozygous, and homozygous mutant heart (upper panel, mitochondria; middle panel, autophagosome; and lower panel, sarcomeric fiber). Scale bar is $1 \mu \mathrm{m}$. $* P<0.05, * * P<0.01$, two-way ANOVA. 
CVR-2018-1173R3

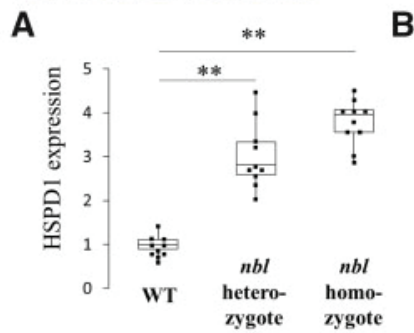

B
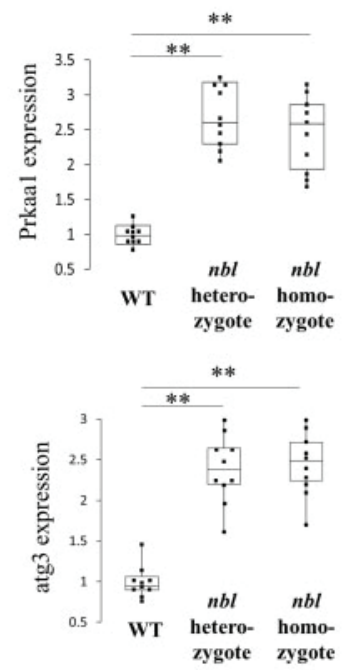
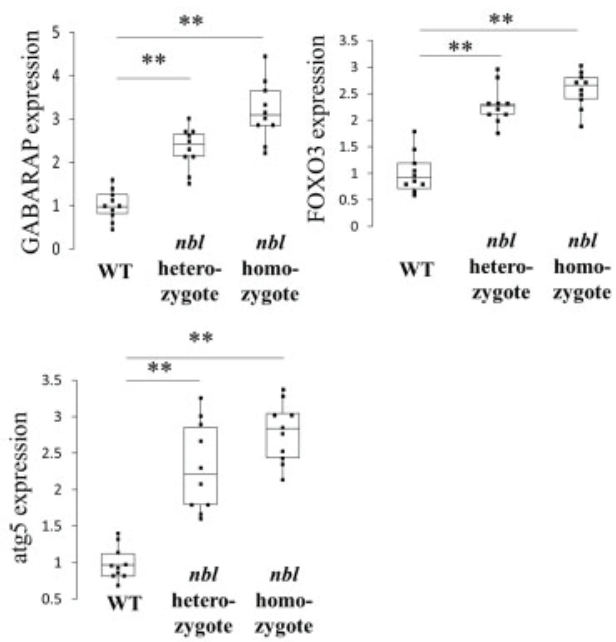

C
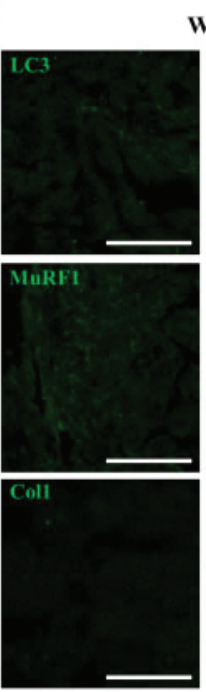

WT
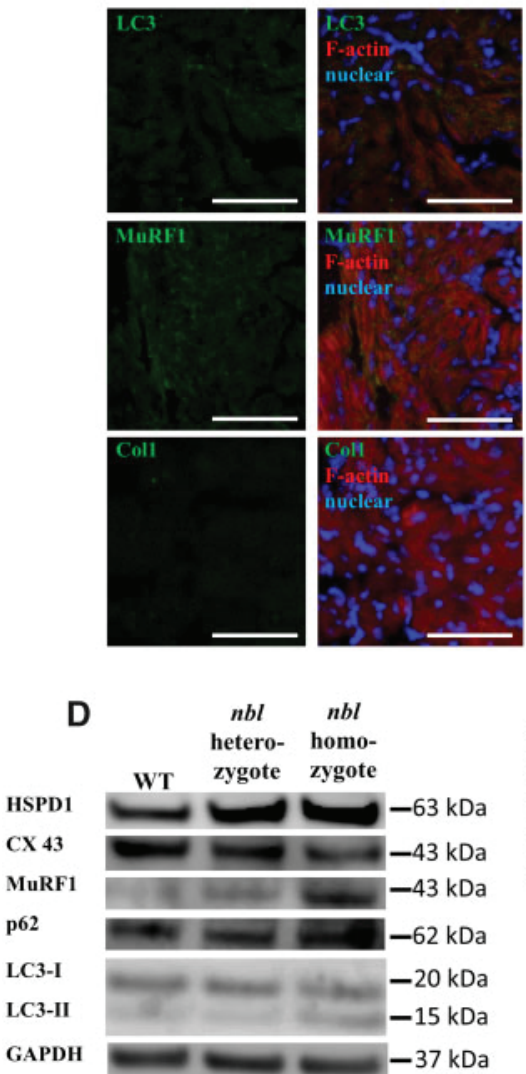
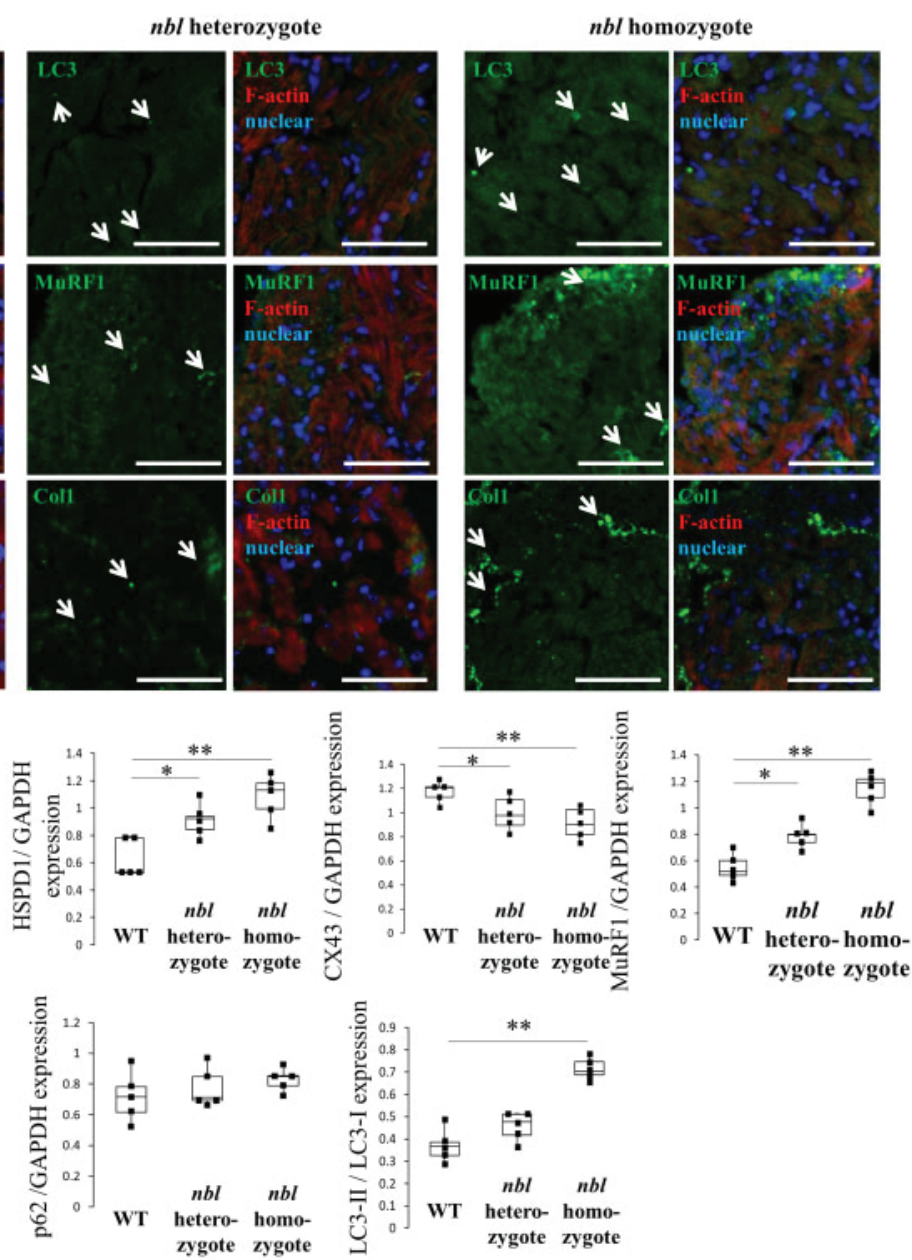

Figure 3 Autophagy was increased in the $n b l$ mutant heart. (A) qPCR analysis for HSPD1, in WT, nbl heterozygous and nbl homozygous mutant heart $(n=10)$. (B) qPCR analysis for autophagy-related genes (Prkaa1, FOXO3, gabarap, Atg3, Atg5) in WT, nbl heterozygous and nbl homozygous mutant hearts $(n=10)$. (C) Immunohistochemistry of autophagy-related proteins (LC3), MuRF1, and collagen1 in WT, nbl heterozygous, and nbl homozygous hearts $(n=5)$. Scale bar is $50 \mu \mathrm{m}$. (D) Protein level of HSPD1, CX43, MuRF1, and LC3 I/II in WT, nbl heterozygous, and nbl homozygous hearts $(n=5)$. Right graphs show concentration of western-blot bands. $* P<0.05$, $* * P<0.01$, two-way ANOVA. 
CVR-2018-1173R3

A

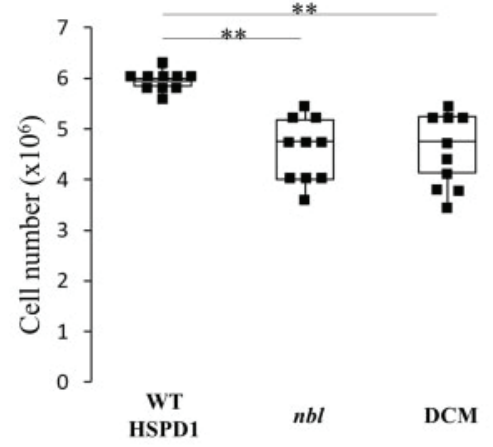

C
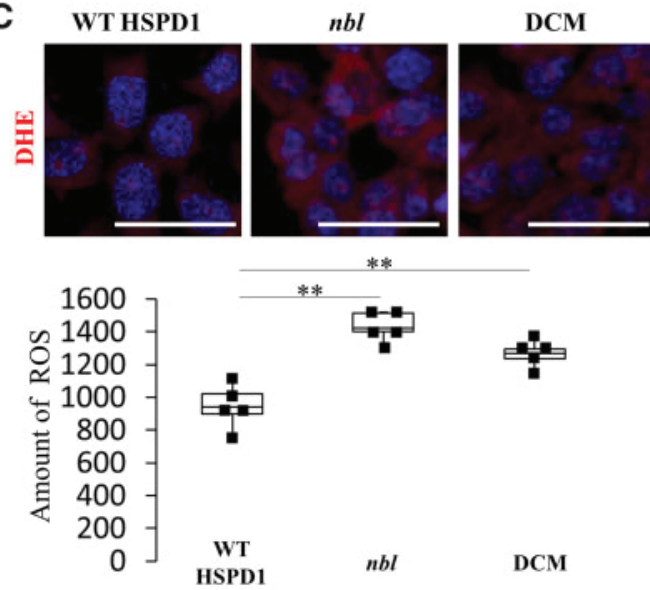

B

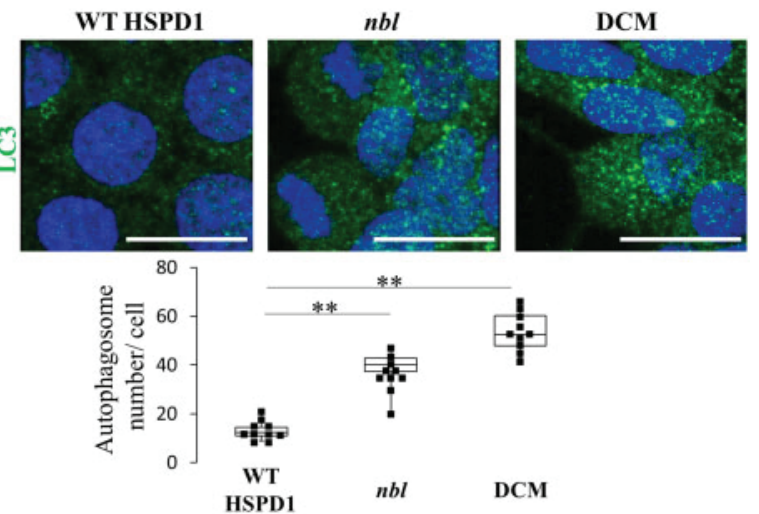

D WT HSPD1
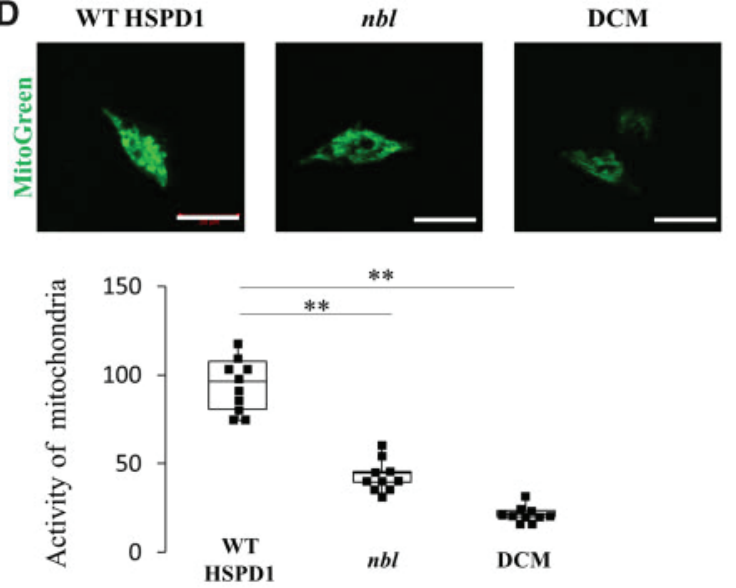

Fission
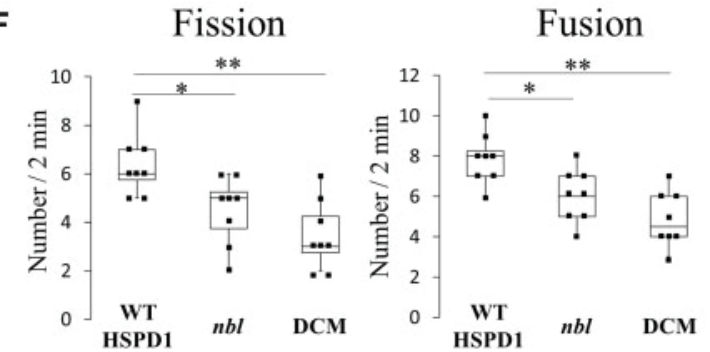

G

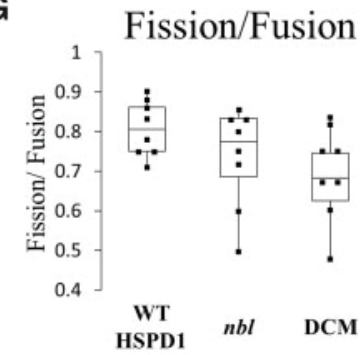

Figure 4 Mitochondrial dysfunction was caused by mutation in HSPD1. (A) Cell count of Flp-In T-REx cells transfected with WT, nbl, and DCM types of HSPD1. $1.0 \times 10^{6}$ cells of each HSPD1-induced cells were incubated for 2 days $(n=10)$. (B) Immunohistochemistry of LC3 in HSPD1-induced cells $(n=10)$. Scale bar is $10 \mu \mathrm{m}$. Lower graph shows the number of autophagosomes. (C) DHE staining in the three HSPD1-expressing cell lines $(n=5)$. Scale bar is $100 \mu \mathrm{m}$. Lower graph shows intensity of fluorescence $(D)$. Mitochondrial staining in the three HSPD1-expressing cell lines. Lower panel shows fluorescence 
intensity $(n=10)$. Scale bar is $100 \mu \mathrm{m}$. Lower graph shows intensity of fluorescence. (E) Mitochondrial fission and fusion cycle in each of the HSPD1-induced cells $(n=5)$. Arrow indicates the two mitochondria fusion and fission in $120 \mathrm{~s}$ of time frame. Scale bar is $5 \mu \mathrm{m}$. (F) Number of fission and fusion cycles in each type of HSPD1-induced cells. Individual data points, box, and whisker plots showing the median, inter-quartile range, and maximum and minimum values $(n=8)$. (G) Fission:fusion ratio in each HSPD1-induced cell $(n=8)$. Individual data points, box, and whisker plots showing the median, inter-quartile range, and maximum and minimum values. $* P<0.05$, $* * P<0.01$, two-way ANOVA.

by restriction fragment length polymorphism (RFLP) (Figure $1 B$ and $C$ ). We performed direct sequencing for 400 healthy Japanese individuals. However, we could not find Thr320Ala missense mutation in any of the normal control DNA. One son had a medical history of arrhythmia, and the other son had been diagnosed with DCM. Both sons suffered sudden death at ages 16 and 21. The daughter of the patient was also diagnosed with DCM and is currently awaiting heart transplantation. The mutation site corresponds to the apical domain of HSPD1(Figure 1D). Multiple species alignment showed high conservation of this domain of the HSPD1protein. ${ }^{19}$ Interestingly, point mutations in $\mathrm{nbl}$ mutant zebrafish (Val324Glu $)^{19}$ and DCM female patients corresponded to the same conserved apical domain, only four amino acids apart, of the HSPD1 protein (Figure $1 E$ ). The amino acid sequence alignment in both the zebrafish and humans showed $79 \%$ sequence identity in the HSPD1 protein, with $88 \%$ sequence identity in its apical domain (Figure $1 E$ ). These results encouraged us to study the role of HSPD1 in the heart using nbl mutant zebrafish.

\subsection{Atrio-ventricular block, dilated heart, and sudden death developed nbl mutants}

The zebrafish were grown at $28.5^{\circ} \mathrm{C}$ (i.e. non-stress conditions). Under optimal conditions, the life span of WT zebrafish is $\sim 2-3$ years. However, nbl homozygous mutant zebrafish do not survive beyond 16 months, with the highest mortality rate being $\sim 12$ months. Similarly, $\mathrm{nbl}$ heterozygous also showed a high mortality rate at $\sim 15$ months (Figure 2A). Furthermore, to check the physiological strength of $\mathrm{nbl}$ mutants, we used a water flow chamber system (see Supplementary material online, Figure S1B and $C$ ) as per the procedure described in the Methods section. Healthy WT fish could easily swim against the stream $(+0.5)$, whereas 5 -month-old $n b l$ homozygous mutants showed reduced exercise tolerance with a score of -1.5. When 8-month-old zebrafish were used, $n b$ l homozygous mutants showed a further lowered exercise tolerance of -8.0, as compared with WT with a score of +4.0. Interestingly, $n b l$ heterozygotes also showed a reduced exercise tolerance of -4.5 (Figure 2B and see Supplementary material online, Movie S1). The difference was not significant at 5-month-old, however, the difference reached statistical significance at 8-month-old. We hypothesized that the loss of physiological strength could be related to cardiac dysfunction. Therefore, we anaesthetized the 8-month-old zebrafish and exposed the heart by dissecting the nearby abdominal skin. While observing under a dissecting microscope, nbl homozygous mutants showed dilated ventricles with increased connective tissue deposition around the heart, as compared with the WT fish (see Supplementary material online, Figure S3). Interestingly, using 5-month-old cmlc2; GFP transgenic zebrafish, we found that $87 \% \mathrm{nbl}$ homozygous zebrafish developed AV block (>6 AV block/min; 13 out of 15). About $46 \% \mathrm{nbl}$ heterozygous zebrafish also showed AV block ( $<3 \mathrm{AV}$ block/min; 7 out of 15) (Figure 20). Histological analysis by haematoxylin and eosin staining showed significantly reduced ventricular wall thickness in 8-month-old $\mathrm{nbl}$ homozygote and heterozygote, as compared with WT fish (Figure 2D). Next, Azan staining of the ventricular chamber showed a higher level of collagen deposition in the nbl homozygotes compared to WT and heterozygotes (Figure 2E, upper panel). Furthermore, DHE staining showed elevated levels of ROS in the nbl homozygotes and heterozygotes when compared with the WT fish (Figure 2E, lower panel). Given that the mitochondria are the major source of ROS production, we analysed the ultrastructure of ventricular cardiomyocytes by transmission electron microscopy. We found that nbl homozygotes had many mitochondria with variable electron density (Figure $2 F$, upper panel). Additionally, mitochondria numbers were increased but mitochondrial size was decreased in nbl homozygous hearts compared to WT and heterozygotes (see Supplementary material online, Figure S4A and B). Interestingly, we found that the number of autophagosomes in $\mathrm{nbl}$ homozygotes was significantly higher than in heterozygotes and completely absent in WT ventricular cardiomyocytes (Figure 2F, middle panel). In addition, the sarcomeric structures were ruptured and thinner in the $n b l$ homozygotes and heterozygotes compared those in WT fish (Figure 2F, lower panel). From these results, we hypothesize that mutation in HSPD1 might decrease cardiac stress tolerance, which leads to mitochondrial dysfunction and increased ROS expression, resulting in AV block and cardiac atrophy.

To confirm if mutations in HSPD1can reduce the cardiac stress tolerance level, we used higher temperatures during embryonic development as a stress-inducing factor. Embryos obtained from a $\mathrm{nbl}$ heterozygous cross were grown 24 -h post-fertilization at $37^{\circ} \mathrm{C}$; we found three different phenotypic embryos based on their growth. Some embryos developed normally, while other embryos showed either an 8- or 12-h delay of embryonic growth (see Supplementary material online, Figure S5A). All of the embryos with normal growth were WT. However, $70 \%$ of embryos with $8 \mathrm{~h}$ of developmental delay, and $80 \%$ of the embryos with $12 \mathrm{~h}$ of growth delay, were $\mathrm{nbl}$ heterozygote and $\mathrm{nbl}$ homozygote, respectively (see Supplementary material online, Figure S5B). We found that at 3.5-day post-fertilization, $48 \%$ (30/62) of nbl homozygous embryos had developed AV block (see Supplementary material online, Figure S5C and D and Movie S2), while 79\% (49/62) of $n b l$ homozygous embryos had developed pericardial oedema (see Supplementary material online, Figure S5E and F). qPCR results showed increased mRNA expression of HSPD1 in heat stress conditions as compared with nonstress conditions, in both nbl homozygous and WT embryos. Also, the mRNA expression level of HSPD1 in nbl homozygous embryos was higher as compared with that in WT in both non-stress and heatstressed conditions (see Supplementary material online, Figure S5G). Western-blot results of the $n b l$ embryos showed that protein expression of HSPD1 was increased in nbl homozygotes as compared to WT fish (see Supplementary material online, Figure $\mathrm{S} 5 \mathrm{H}$ ). These results confirm that mutations in HSPD1 led to the development of AV block and pericardial oedema in $n b l$ homozygous embryos under stress conditions.

\subsection{Autophagy-related genes expression in- creased in $\mathrm{nbl}$ homozygous mutant hearts}

HSPD1 increased in a similar pattern (Figure 3A). Since we found autophagosomes in $\mathrm{nbl}$ homozygous hearts by TEM analysis (Figure 2F), we 
CVR-2018-1173R3

A
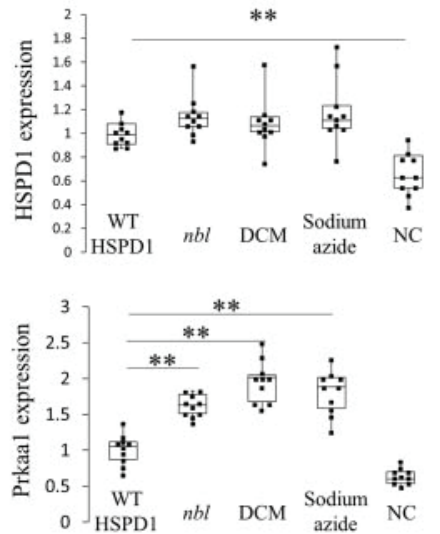

B
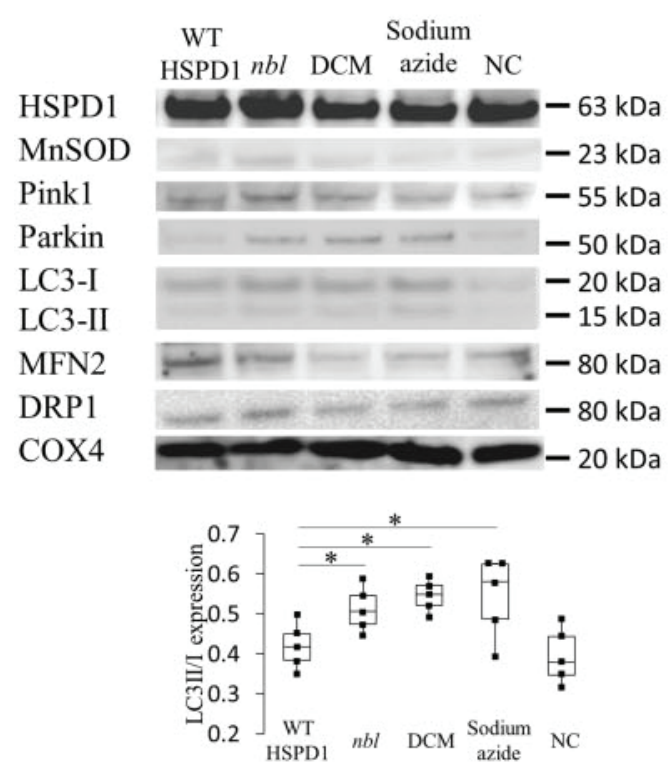

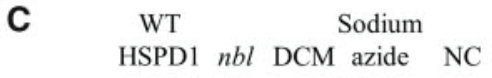

p62

$--62 \mathrm{kDa}$

LC3-I $-20 \mathrm{kDa}$

LC3-II

COX 4

$-15 \mathrm{kDa}$

D
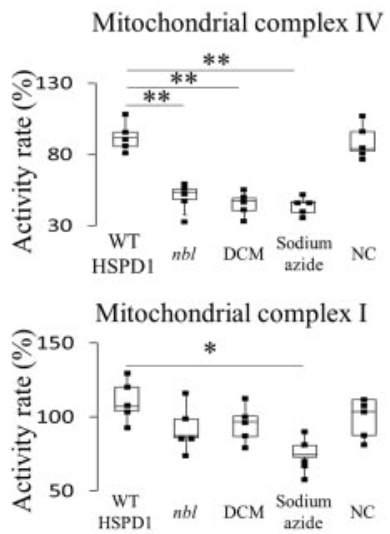
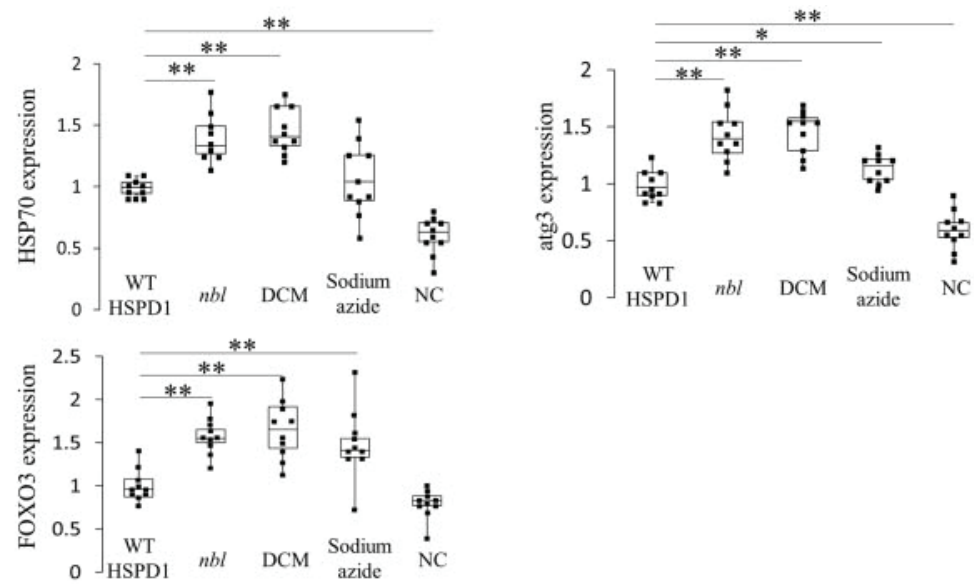

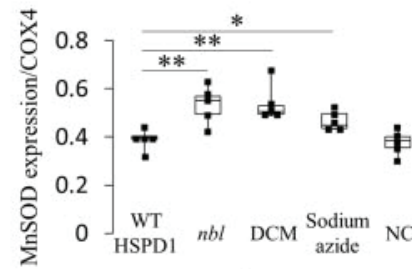

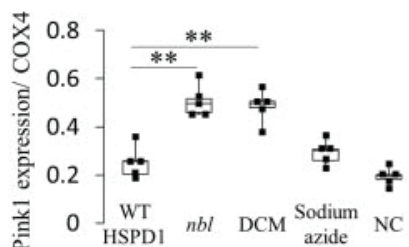
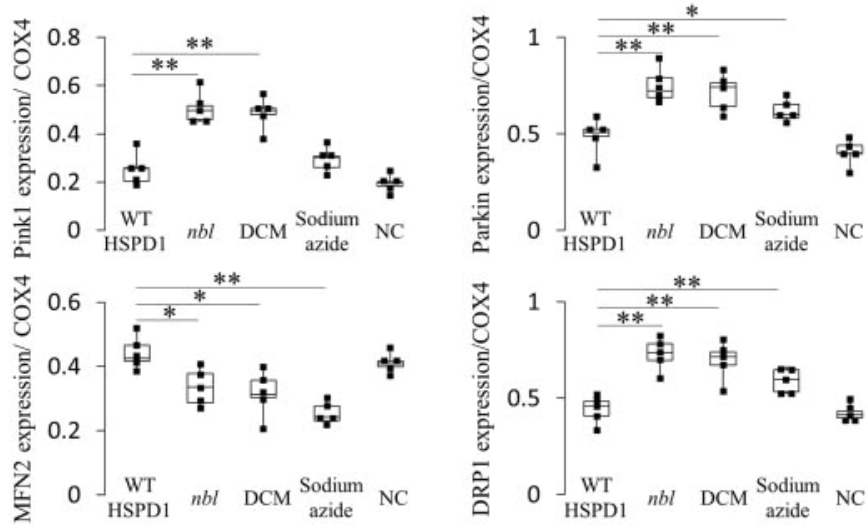
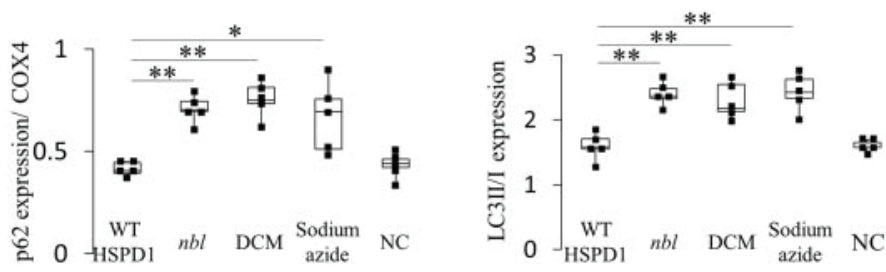

Mitochondrial complex III

E
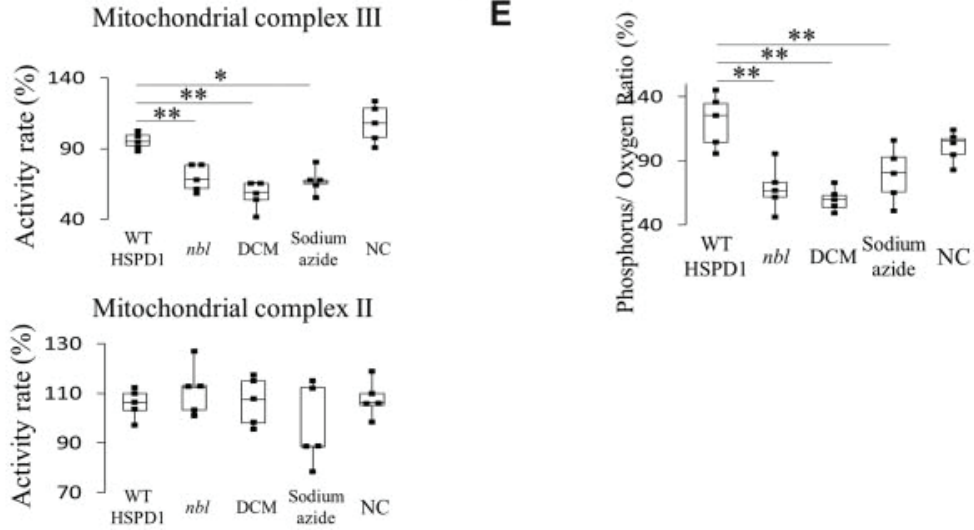
assumed that autophagy might induce cardiac atrophy in $n b$ homozygous mutants. Therefore, to understand its mechanistic basis, we analysed expression patterns of autophagy-related genes in the heart of 8-monthold adult WT and $n b l$ homozygotes. mRNA expression levels of autophagy inducer, Prkaa1 (AMP kinase), and FOXO3 were significantly increased in both $n b l$ heterozygous and homozygous fish. Furthermore, autophagy-related genes GABARAP (atg8), atg5, and atg3 also showed higher expression in $n b l$ heterozygous and homozygous fish (Figure 3B). Further, immunohistochemical analysis showed an increased expression of MuRF1 and collagen (Col1) in $n b l$ heterozygotes and homozygotes as compared to WT fish (Figure 3C, middle and lower panels). Microtubule-associated protein 1 A/1B-light chain 3 (LC3) is a soluble protein with a molecular mass of $\sim 17 \mathrm{kDa}$. The cytosolic form of LC3 (LC3-I) conjugates to phosphatidylethanolamine and form LC3phosphatidylethanolamine conjugate (LC3-II), which is recruited to autophagosomal membranes. ${ }^{23}$ The immunohistochemical analysis showed that the expression of LC3 was higher in nbl homozygotes compared to nbl heterozygotes. However, the LC3 expression was absent in WT (Figure 3C, upper panel). Western-blot data showed that LC3 II/I was significantly higher in $n b l$ homozygotes than in heterozygotes and WT. We also analysed protein expression levels of p62 in WT and mutants (Figure 3D). Thus, these results, along with the pattern of deaths of $n b l$ mutants, indicate that autophagy was induced in an age-dependent manner, and thus $\mathrm{nbl}$ homozygous fish showed higher death rates at 78 months after birth. We also tried to analyse the p62 level using western blotting. However, the expression level of p62 was similar in WT, heterozygotes, and homozygotes. It is known that p62 is continuously degraded once incorporated into the autophagosome. Therefore, detecting and correlating the p62 level and autophagic flux without the use of autophagic inhibitors like bafilomycin may have resulted in a similar pattern in WT and mutants. Western-blot results of the $n b l$ heart showed that protein expression of HSPD1,and MuRF1 was increased in $\mathrm{nbl}$ homozygotes and heterozygotes as compared with in WT fish (Figure 3D). Mutation in HSPD1 stimulates the HSPD1 expression to enhance a stress response. These results suggest that although physiological stress resulted in increased HSPD1 expression, the point mutation resulted in loss of function of HSPD1, and thus in higher autophagy in $\mathrm{nbl}$ homozygotes and heterozygotes. Connexin 43 (CX43) is important for inter-cardiomyocyte junctions and is known to regulate synchronized cardiac beating in vitro. Interestingly, the protein level of CX43 was decreased in $n b l$ homozygous and heterozygous hearts, which might cause arrhythmia.

\subsection{Induction of mutant HSPD1 increased autophagy and decreased mitochondrial function}

Using the Flp-In T-REx system, we created stable transfected HEK293 cell lines, which express HSPD1 genes of the WT and $n b l$ and DCM types (see Methods section). The gene expression was induced by tetracycline treatment (see Supplementary material online, Figure S6). Each stable cell line was cultured in $10 \%$ DMEM medium containing $1 \mu \mathrm{g} / \mathrm{ml}$ of tetracycline on day $0\left(1.0 \times 10^{6}\right.$ cells). After 2 days of incubation, the average cellular proliferation in $n b$ type $\left(4.6 \times 10^{6}\right.$ cells $)$ and DCM $\left(4.9 \times 10^{6}\right.$ cells $)$ type was significantly lower than in WT $\left(6.0 \times 10^{6}\right.$ cells, Figure $\left.4 A\right)$. The nbl (35.4 autophagosomes) and DCM (53.4 autophagosomes) transfected cells showed higher expression of autophagic markers as compared with the WT (10.8 autophagosomes) transfected cells by the immunohistochemistry for LC3 (Figure 4B). Since we found high ROS and irregular electron density of mitochondria in nbl mutants, we examined if similar patterns are obtained in vitro. DHE staining showed elevated levels of ROS in nbl and DCM cell lines, as compared with WT cell lines (Figure 4C). Therefore, we used MitoGreen (TaKaRa Bio Inc., Kusatsu, Japan) dye to detect the activity of mitochondria using confocal laser microscopy. The expression of MitoGreen was significantly lower in the $n b l$ and DCM cell lines, as compared with in WT cell lines (Figure 4D). We assumed that HSPD1 chaperone activity was decreased in mutant cell lines. Therefore, we analysed HSP60/HSP10 folding activity in each of the HSPD1-induced cell lines. We found that folding activity of HSP60/HSP10 was lower in the mutant cell lines (see Supplementary material online, Figure S7). Furthermore, when we observed mitochondrial activity using time-lapse microscopy, we found that the fission and fusion cycles of mitochondria in $\mathrm{nbl}$ (fission: 5.0/2 min, fusion 6.0/2 min) and DCM cell lines (fission: $3.0 / 2 \mathrm{~min}$, fusion: $4.5 / 2 \mathrm{~min}$ ) were significantly lower than those of the WT cell lines (fission: 6.0/2 min, fusion: 8.0/ 2 min; Figure 4E and F and see Supplementary material online, Movie S3). Interestingly the fission:fusion ratios were similar in all three cell lines (Figure $4 G$ ). We performed similar experiments in $\mathrm{H} 9 \mathrm{C} 2$ cell lines(a myogenic cell line derived from embryonic rat heart ventricles)overexpressed with WT, nbl-, and DCM types of HSPD1. Fission and fusion were decreased in $\mathrm{H} 9 \mathrm{C} 2$ cell lines overexpressing $n b l-$ and DCM types of HSPD1 compared to $\mathrm{H} 9 \mathrm{C} 2$ cell lines overexpressing WT type of HSPD1 as it is shown in Supplementary material online, Figure S8 (see Supplementary material online, Movie S4):

\subsection{Mitophagy was induced by decrease in mitochondrial complexes III and IV activity}

We investigated whether the decrease in mitochondrial activity caused an increase in mitophagy. We used sodium azide, a potent inhibitor of complex IV, as a positive control in our experiment. qPCR results demonstrated a significant increase in Prkaa1 (AMP kinase), FOXO3, and atg3 mRNA expression in HSPD1-nbl and HSPD1-DCM cell lines (Figure 5A). Western-blot results of the mitochondrial extract show that protein expression of the genes involved in autophagy, MnSOD, Pink1, Parkin, and LC3-II were increased in HSPD1-nbl and HSPD1-DCMinduced HEK293 cells, as compared with WT-induced HEK293 cells (Figure 5B). Parkin and Pink1 are located in mitochondria and trigger mitochondrial autophagy, which is known as mitophagy. It was confirmed that mitochondria-specific autophagy (mitophagy) occurred by inhibiting

Figure 5 Mutation in HSPD1 caused decrease in mitochondrial activity. (A) qPCR analysis for HSPs (HSPD1, HSPA5), Prkaa1, FOXO3, atg3 genes in the three HSPD1-expressing cell lines $(n=10)$. (B) Western-blot analysis for mitochondria of the three HSPD1-expressing cell lines. Sodium azide used as positive control for mitochondrial complex IV dysfunction $(n=5)$. Graphs show concentration of western-blot bands. (C) Flux assay for mitochondrial autophagy-related proteins $(n=5)$. Lower graph shows concentration of western-blot bands. (D) Mitochondrial complex activities in the three HSPD1-expressing cell lines $(n=5)$. (E) Phosphorus/oxygen analysis for WT and nbl- and DCM-type HSPD1 induced cell lines $(n=5)$. NC, negative control; untreated HEK293 cell. $* P<0.05, * * P<0.01$, two-way ANOVA. 
CVR-2018-1173R3

A

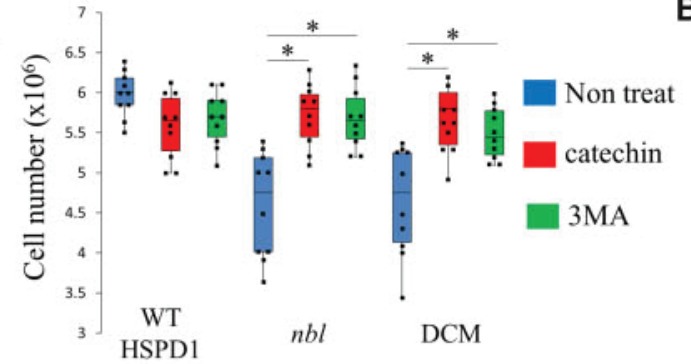

C

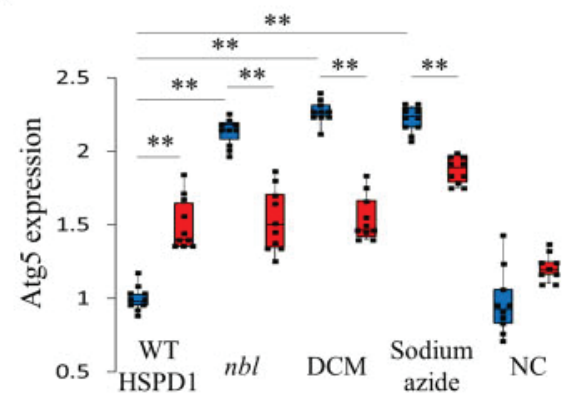

E

catechin $\frac{\text { HSPD1 }}{-} \frac{n b l}{-+} \frac{\mathrm{DCM}}{-+} \frac{\mathrm{NC}}{-+}$
MuRF1
GAPDH

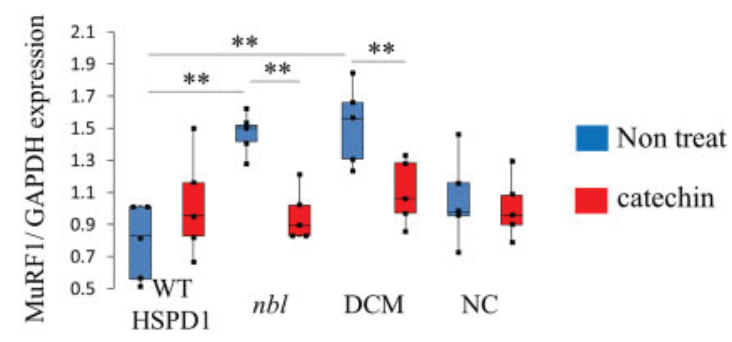

G

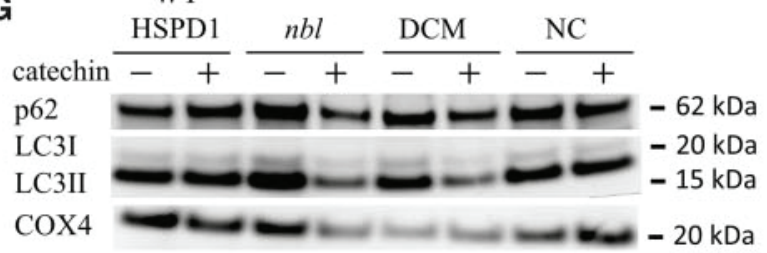

B

D
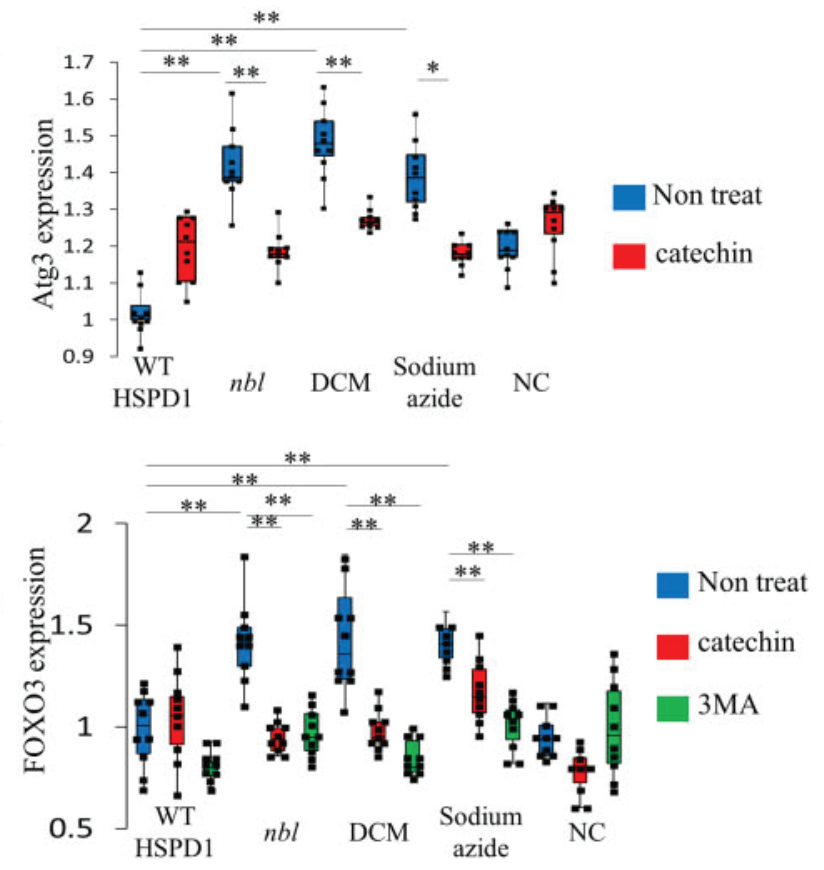

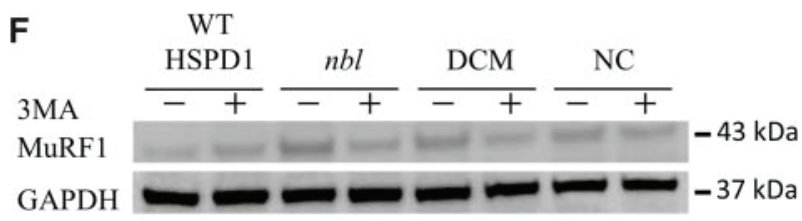

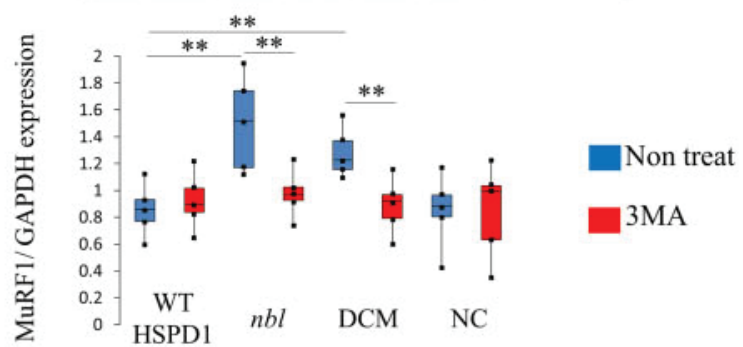

H

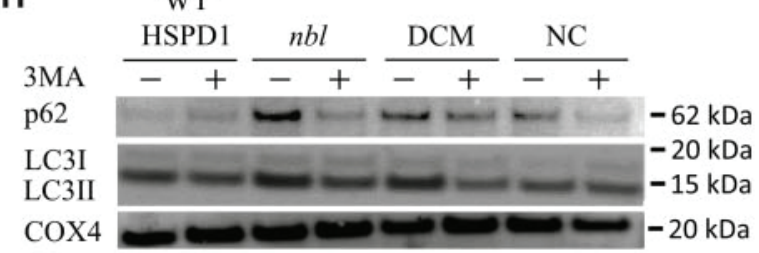

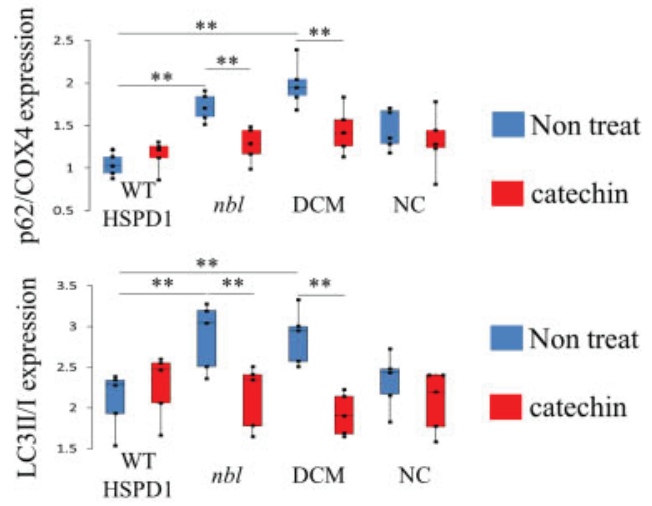
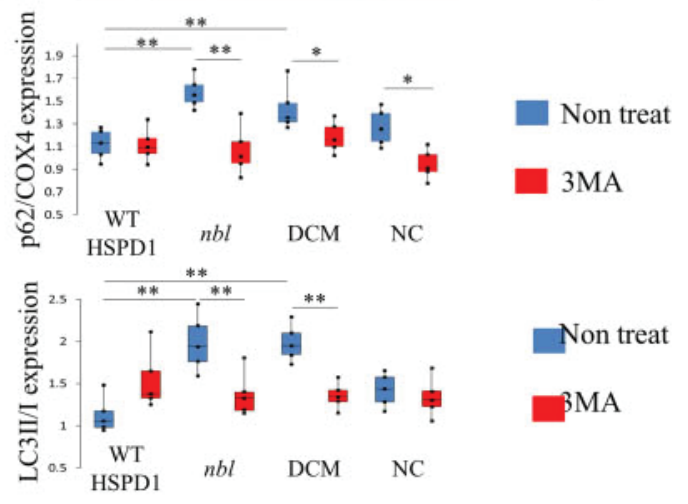
Figure 6 Inhibition of ROS and autophagosome activity rescued FOXO3 and MuRF1 expression. (A) Cell number of HSPD1-induced cell lines treated with catechin or 3MA $(n=10)$. (B) mRNA level of atg3 in HSPD1-induced cell lines treated with catechin $(n=10)$. (C) mRNA level of atg5 in HSPD1-induced cell lines treated with catechin $(n=10)$. (D) mRNA level of FOXO3 in HSPD1-induced cell lines treated with 3MA and catechin $(n=10)$. (E) Protein level of MuRF1 in overexpression of HSPD1 H9C2 cell lines treated with catechin. Lower graph is concentration of western-blot band $(n=5)$. $(F)$ Protein level of MuRF1 in overexpression of HSPD1 H9C2 cell lines treated with 3MA. Lower graph is concentration of western-blot band $(n=5)$. ( $($ ) Protein level of p62 and LC3 II/I in HSPD1overexpressed mitochondria in H9C2 cell lines treated with catechin. Lower graphs show concentration of western-blot bands $(n=5)$. $(H)$ Protein level of $\mathrm{p} 62$ and LC3 II/I in HSPD1-overexpressed mitochondria in H9C2 cell lines treated with 3MA. Lower graphs show concentration of western-blot bands $(n=5)$. NC, negative control; untreated H9C2 cell. $* P<0.05$, **P<0.01, two-way ANOVA.

the lysosome, and that expression of LC3-II and P62 was increased in mutant HSPD1-induced cell lines (Figure 5C and see Supplementary material online, Figure S9). Protein levels of mitofusin (MFN2) and mitofission (Drp1) were decreased in HSPD1-nbl- and HSPD1-DCM-induced HEK293 cells, indicating a decrease in mitochondrial fusion. Interestingly, mRNA and protein expression patterns of autophagy-related genes in HSPD1-nbl and HSPD1-DCM-induced HEK293 cells were similar to sodium azide-treated HEK293 cells. This encouraged us to check if the increase in autophagy in HSPD1-nbl and HSPD1-DCM-induced HEK293 cells was due to a decrease in activity of any mitochondrial complex. Subsequently, we found that the activity of mitochondrial complex IV was significantly reduced in both HSPD1-nbl and HSPD1-DCM-induced HEK293 cells, similar to sodium azide-treated cells. The activity of complex III was also decreased in HSPD1-nbl and HSPD1-DCM-induced HEK293 cells; however, the activity rate of complexes I and II were similar (Figure 5D). Furthermore, we performed phosphorus/oxygen (P: O) analysis in each of the HSPD1-induced cell lines. P:O ratios decreased in the mutant cell lines (Figure 5E).

\subsection{Inhibition of ROS and autophagosome formation rescued FOXO3 and MuRF1 expression}

Next, we performed rescue experiment, in which we used catechin and 3-methyladenine (3MA) to inhibit ROS expression and autophagosome formation, respectively, in HSPD1-induced HEK293 cell lines. Both, catechin and 3 MA, rescued cell division in $n b l$ and DCM type HEK cell line (Figures 4A and 6A). Further, we found that catechin treatment resulted in decreased mRNA expression levels of atg3 and atg5 in nbl- and DCMtype HSPD1-induced HEK293 cell lines, as compared with respective non-treated cell lines (Figure 6B and C). To establish if mitophagy contributes to DCM type phenotype, we compared the change of expression level of $\mathrm{FOXO} 3$ under catechin and 3MA treatment in three types of HSPD1 induced HEK293 cell lines. We found that mRNA expression level of FOXO3 in nbl- and DCM-type HSPD1 cell lines under catechin and $3 \mathrm{MA}$ treatment was significantly decreased when compared with non-treated corresponding cell line (Figure 6D). We, then, used HSPD1overexpressed $\mathrm{H} 9 \mathrm{C} 2$ cell line, for the rescue experiment. We found that inhibition of ROS production and autophagosome formation lead to decreased expression of MuRF1 in nbl- type and DCM-type HSPD1 cell line (Figure 6E and F). Furthermore, in mutant HSPD1-expressed cells, p62 and LC3 II/I ratios were decreased upon treatment with catechin and $3 \mathrm{MA}$. We investigated whether the phenotype of $n b l$ zebrafish can be rescued by catechin and 3MA treatment. We found that cardiac oedema and the AV-block rate in untreated zebrafish (NC) were higher than in zebrafish treated with catechin and 3MA (see Supplementary material online, Figure S10). These experiments established that inhibition of mitophagy leads to decrease in expression level of FOXO3 and MuRF1 suggesting direct involvement of mitophagy in cardiac atrophy.

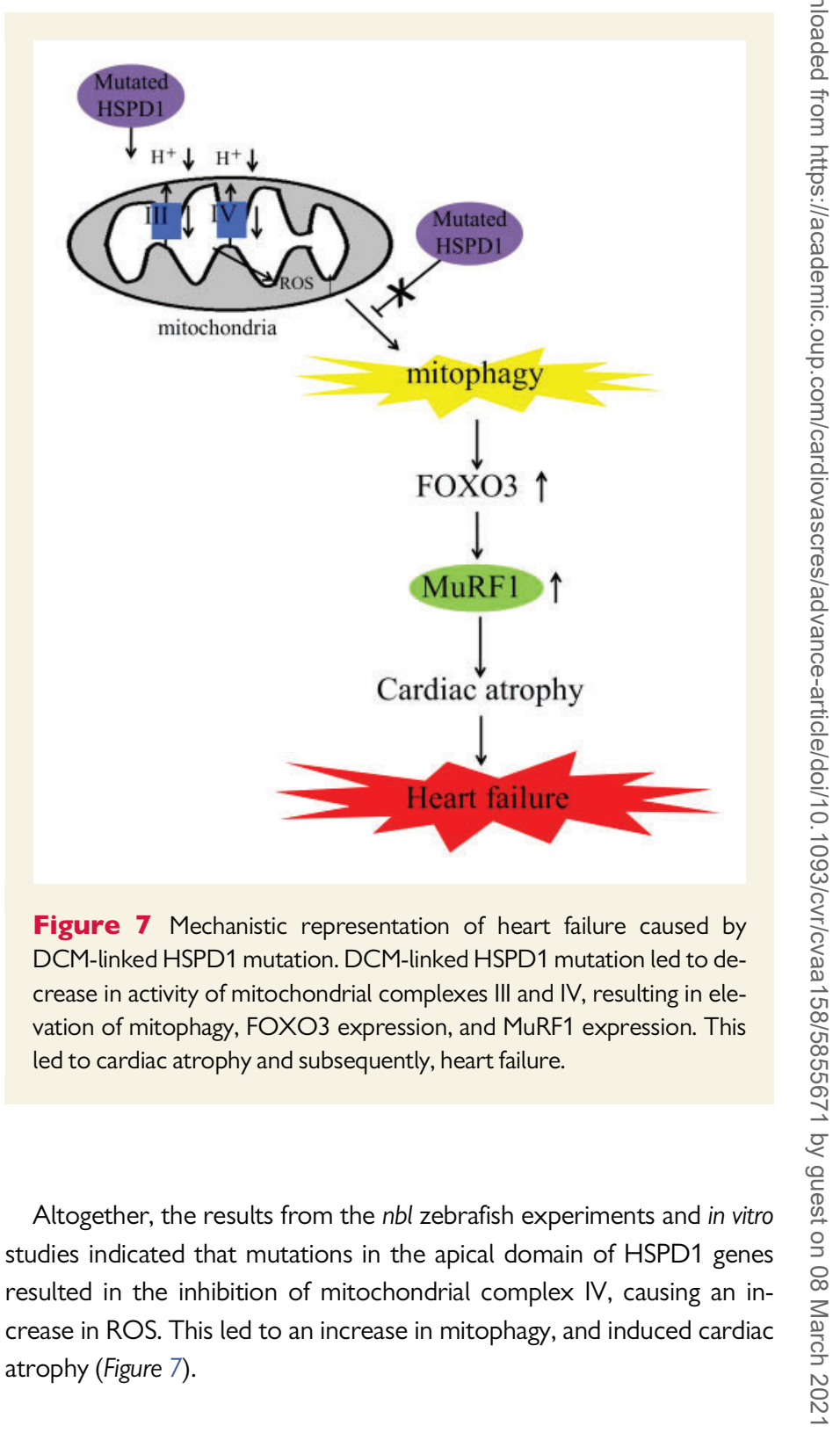

\section{Discussion}

In a previous study, Makino et al. ${ }^{19}$ developed nbl mutants that lacked regenerative capacity and showed pericardial oedema under heat stress. The presence of a point mutation in the apical domain of HSPD1 in a familial DCM patient, and a similar mutation in a zebrafish $\mathrm{nbl}$ mutant, has prompted great interest in studying the mechanistic basis of the role of HSPD1 in cardiac disease. In this study, we demonstrated that the upregulation of HSPD1 protein, due to a point mutation, inhibited the 
activity of mitochondrial complex IV, therefore resulting in an increase in ROS concentration. High ROS led to significant increase in mitophagy in cardiac tissue, causing heart failure (Figure 7).

HSPD1 is an evolutionary conserved ubiquitously expressed protein. The major functions of HSPD1 are to maintain the integrity of cellular proteins, particularly in response to environmental changes; ${ }^{24}$ control the transport and maintenance of mitochondrial proteins, ${ }^{25}$ and assist in the replication of mitochondrial DNA. ${ }^{26}$ Mutation in HSPD1 may cause phenotypic changes in high HSPD1-expressed organs, such as the heart and brain. ${ }^{27,28}$ In fact, there is a report that HSPD1-mutated patients have exhibited brain hypomyelination and leukodystrophy. ${ }^{10,11} \mathrm{Here}$, we identified the point mutation, Thr320Ala, in exon 8 of HSPD1 gene in one of the familial DCM patients.

DCM is a rare cardiac disease that often leads to heart failure and sudden cardiac death caused by the failure of cardiac conduction systems. ${ }^{29}$ DCM is also associated with excessive proliferation of cardiac fibroblasts. ${ }^{30} \mathrm{nbl}$ mutants at the adult stage showed typical DCM-like phenotypes (i.e. dilation of ventricular chamber, decrease in thickness of ventricular wall, AV block, and cardiac fibrosis) (Figure 2 and see Supplementary material online, Figure S3). However, the mutations in HSPD1 did not affect the skeletal muscle systems in the nbl mutants (data not shown). Therefore, we assume that cardiac dysfunction is the primary cause of sudden death in the nbl mutants. HSPD1 is a stressresponsive gene, and its expression increases to neutralize the physiological responses from any type of stress. ${ }^{31}$ However, nbl mutants showed higher expression of HSPD1 and a subsequent increase in ROS production, even at optimum growth conditions. Since every organism, during its development and growth, faces various forms of stress, we show that a missense mutation in the apical domain of the HSPD1 gene has resulted in functional inactivation of the HSPD1 protein, leading to a continuous increase in ROS around the 7- to 8-month point in $\mathrm{nbl}$ adult fish, thus causing early aging. Therefore, missense mutation of HSPD1 did not affect its stress-responsive expression but, unable to neutralize the physiological response of heat stress.

Since the HSPD1 is a mitochondrial chaperonin, and ROS level is moderated through mitochondria, it is reasonable to assume that any cardiac damage that occurs is initiated through mitochondrial dysfunction. $^{32-34}$ TEM analysis of ventricular chambers of $n b l$ mutants showed mitochondria with variable electron densities, as compared with the mitochondria that exhibited similar electron densities in WT. Interestingly, the mitochondria in $\mathrm{nbl}$ showed a significantly higher number of autophagosome-like structures, suggesting that mitophagy may have some role in the development of DCM-like phenotypes in $\mathrm{nbl}$ mutants.

Mitochondria constantly undergo fission and fusion to generate healthy mitochondria. ${ }^{35}$ Meanwhile, defective mitochondria are eliminated through a form of autophagy called mitophagy. ${ }^{36}$ The stable HEK293 cells, which overexpressed the $n b l$ and DCM types of HSPD1, showed a significant decrease in mitochondrial fission and fusion. Furthermore, we found that nbl type and DCM type HEK293 cells demonstrated a significant decrease in complex IV activity. These results indicate that a point mutation in the apical domain of HSPD1 induced mitochondrial dysfunction.

It was earlier established that the knockdown of HSPD1 resulted in a decrease in complex IV activity. ${ }^{25}$ Autophagy is required for normal development. However, excessive autophagy may cause tissue damage. ${ }^{37}$ In order to understand, if excessive mitophagy is caused by missense mutation in HSPD1, we transfected nbl type and DCM type HSPD1 in HEK293 cell line. In nbl mutants, we found a significant increase in autophagy as compared with WT. MuRF1 was reported to be induced by $\mathrm{FOXO} 3$, and was found in developed cardiac atrophy. ${ }^{38,39}$ The aberrant autophagic response resulted in cardiac damage (MuRF1 expression) in nbl mutants (Figure 3C).

In summary, point mutation in apical domain of HSPD1 led to mitochondrial dysfunction through ROS-mediated increased mitophagy, resulting in a DCM like condition. Therefore, we suggest that HSPD1 is one of the mediators of development of heart failure.

\section{Supplementary material}

Supplementary material is available at Cardiovascular Research online.

\section{Acknowledgements}

We thank Yasushi Kawata of the Department of Chemistry and Biotechnology (Tottori University) for making the ribbon drawing structure of HSPD1. We also thank Taishi Sasaoka, Ichiro Watanabe of Internal Cardiology (Kitasato University) and Takeharu Hayashi of Molecular Pathogenesis (Tokyo Medical and Dental University) for giving patient data. We are grateful to James Thomas (Keio University School of Medicine) for critical reading.

\section{Conflict of interest: none declared.}

\section{Funding}

This study was supported in part by the programme for Grant-in-Aid for Scientific Research (21390248, 22390157, 23132507, 23659414, 19590832, $16 \mathrm{H} 05305,25293181$, and $16 \mathrm{H} 05296)$ from the Ministry of Education, Culture, Sports, Science and Technology of Japan, a research grant for Idiopathic Cardiomyopathy from the Ministry of Health, Labor and Welfare. This study was supported by the Joint Usage/Research Program of Medical Research Institute, Tokyo Medical and Dental University.

\section{References}

1. Schaper J, Froede R, Hein S, Buck A, Hashizume H, Speiser B, Friedl A, Bleese N. Impairment of the myocardial ultrastructure and changes of the cytoskeleton in dilated cardiomyopathy. Circulation 1991;83:504-514.

2. Kimura A. Contribution of genetic factors to the pathogenesis of dilated cardiomyopathy: the cause of dilated cardiomyopathy: genetic or acquired? (genetic-side). Circ J 2011;75:1756-1765.

3. Yoshikawa T. Contribution of acquired factors to the pathogenesis of dilated cardiomyopathy: the cause of dilated cardiomyopathy: genetic or acquired? (acquired-side). Circ 2011;75:1766-1773.

4. Vogel B, Meder B, Just S, Laufer C, Berger I, Weber W, Katus HA, Rottbauer W. Invivo characterization of human dilated cardiomyopathy genes in zebrafish. Biochem Biophys Res Commun 2009;390:516-522.

5. Kimura A. Molecular genetics and pathogenesis of cardiomyopathy. J Hum Genet 2016;61:41-50.

6. Lakdawala NK, Givertz MM. Dilated cardiomyopathy with conduction disease and arrhythmia. Circulation 2010;122:527-534.

7. Keller H, Finsterer J, Steger C, Wexberg P, Gatterer E, Khazen C, Stix G, Gerull B, Höftberger R, Weidinger F. Novel c.367_369del LMNA mutation manifesting as severe arrhythmias, dilated cardiomyopathy, and myopathy. Heart Lung 2012;41: 382-386.

8. Lin KM, Lin B, Lian IY, Mestril R, Scheffler IE, Dillmann WH. Combined and individual mitochondrial HSP60 and HSP10 expression in cardiac myocytes protects mitochondrial function and prevents apoptotic cell deaths induced by simulated ischemia-reoxygenation. Circulation 2001;103:1787-1792.

9. Tang H, Chen Y, Liu X, Wang S, Lv Y, Wu D, Wang Q, Luo M, Deng H. Downregulation of HSP60 disrupts mitochondrial proteostasis to promote tumorigenesis and progression in clear cell renal cell carcinoma. Oncotarget 2016;7: 38822-38388. 
10. Magnoni R, Palmfeldt J, Christensen JH, Sand M, Maltecca F, Corydon TJ, West M, Casari G, Bross P. Late onset motoneuron disorder caused by mitochondrial HSP60 chaperone deficiency in mice. Neurobiol Dis 2013;54:12-23.

11. Magen D, Georgopoulos C, Bross P, Ang D, Segev Y, Goldsher D, Nemirovski A, Shahar E, Ravid S, Luder A, Heno B, Gershoni-Baruch R, Skorecki K, Mandel H. Mitochondrial HSP60 chaperonopathy causes an autosomal-recessive neurodegenerative disorder linked to brain hypomyelination and leukodystrophy. Am J Hum Genet 2008;83:30-42.

12. Sidorik L, Kyyamova R, Bobyk V, Kapustian L, Rozhko O, Vigontina O, Ryabenko D, Danko I, Maksymchuk O, Kovalenko VN, Filonenko VV, Chaschin NA. Molecular chaperone, HSP60, and cytochrome P450 2E1 co-expression in dilated cardiomyopathy. Cell Bio Int 2005;29:51-55.

13. Hara T, Nakamura K, Matsui M, Yamamoto A, Nakahara Y, Suzuki-Migishima R, Yokoyama M, Mishima K, Saito I, Okano H, Mizushima N. Suppression of basal autophagy in neural cells causes neurodegenerative disease in mice. Nature 2006;441: 885-889.

14. Chen MC, Chang JP, Wang YH, Liu WH, Ho WC, Chang HW. Autophagy as a mechanism for myolysis of cardiomyocytes in mitral regurgitation. Eur J Clin Invest 2011;41: 299-307.

15. Wang L, Hao H, Wang J, Wang $X$, Zhang S, Du Y, Lv T, Zuo L, Li Y, Liu H. Decreased autophagy: a major factor for cardiomyocyte death induced by $\beta 1$-adrenoceptor autoantibodies. Cell Death Dis 2015;6:e1862.

16. Nishida K, Kyoi S, Yamaguchi O, Sadoshima J, Otsu K. The role of autophagy in the heart. Cell Death Differ 2009;16:31-38.

17. Kubli DA, Gustafsson $A B$. Mitochondria and mitophagy: the yin and yang of cell death control. Circ Res 2012;111:1208-1207.

18. Narendra D, Tanaka A, Suen DF, Youle RJ. Parkin is recruited selectively to impaired mitochondria and promotes their autophagy. J Cell Biol 2008;183:795-803.

19. Makino S, Whitehead GGc, Lien CL, Kim S, Jhawar P, Kono A, Kawata Y, Keating MT. Heat-shock protein 60 is required for blastema formation and maintenance during regeneration. Proc Natl Acad Sci U S A 2005;102:14599-14604.

20. Arimura T, Takeya R, Ishikawa T, Yamano T, Matsuo A, Tatsumi T, Nomura T, Sumimoto $\mathrm{H}$, Kimura A. Dilated cardiomyopathy-associated FHOD3 variant impairs the ability to induce activation of transcription factor serum response factor. Circ J 2013;77:2990-2996.

21. Adisa AO, Udeabor SE, Kubesch A, Barbeck M, Ghanaati S. The utility of azan trichrome staining in ameloblastoma. Niger Postgrad Med J 2016;23:44-46.

22. Kanda Y. Investigation of the freely-available easy-to-use software "EZR" (Easy R) for medical statistics. Bone Marrow Transplant 2013;48:452-458.

23. Kabeya Y, Mizushima N, Ueno T. LC3, a mammalian homologue of yeast Apg8p, is localized in autophagosome membranes after processing. EMBO J 2000;19: 5720-5728.

24. Radford SE. GroEL: more than just a folding cage. Cell 2006;125:831-833.
25. Kim KH, Song K, Yoon SH, Shehzad O, Kim YS, Son JH. Rescue of PINK1 nullspecific mitochondrial complex IV deficits By ginsenoside Re activation of nitric oxide signaling. J Biol Chem 2012;287:44109-44120.

26. Kaufman BA, Kolesar JE, Perlman PS, Butow RA. A function for the mitochondrial chaperonin HSP60 in the structure and transmission of mitochondrial DNA nucleoids in Saccharomyces cerevisiae. J Cell Biol 2003;163:457-461.

27. Barone R, Macaluso F, Sangiorgi C, Campanella C, Marino Gammazza A, Moresi V, Coletti D, Conway de Macario E, Macario AJ, Cappello F, Adamo S, Farina F, Zummo G, Di Felice V. Skeletal muscle Heat shock protein 60 increases after endurance training and induces peroxisome proliferator-activated receptor gamma coactivator $1 \propto 1$ expression. Sci Rep 2016;6:19781.

28. Noelker C, Morel L, Osterloh A, Alvarez-Fischer D, Lescot T, Breloer M, Gold M, Oertel WH, Henze C, Michel PP, Dodel RC, Lu L, Hirsch EC, Hunot S, Hartmann A. Heat shock protein 60: an endogenous inducer of dopaminergic cell death in Parkinson disease. J Neuroinflammation 2014;11:86-98.

29. Norton N, Li D, Rieder MJ, Siegfried JD, Rampersaud E, Züchner S, Mangos S, Gonzalez-Quintana J, Wang L, McGee S, Reiser J, Martin E, Nickerson DA, Hershberger RE. Genome-wide studies of copy number variation and exome sequencing identify rare variants in BAG3 as a cause of dilated cardiomyopathy. Am J Hum Genet 2011:88:273-282.

30. Pluess M, Daeubler G, Dos Remedios CG, Ehler E. Adaptations of cytoarchitecture in human dilated cardiomyopathy. Biophys Rev 2015;7:25-32.

31. Kreutmayer SB, Messner B, Knoflach M, Henderson B, Niederegger H, Böck G, Van der Zee R, Wick G, Bernhard D. Dynamics of heat shock protein 60 in endothelial cells exposed to cigarette smoke extract. J Mol Cell Cardiol 2011;51:777-780.

32. Kao TY, Chiu YC, Fang WC, Cheng CW, Kuo CY, Juan HF, Wu SH, Lee AY. Mitochondrial Lon regulates apoptosis through the association with Hsp60-mtHsp70 complex. Cell Death Dis 2015;6:e1642.

33. Chen Y, Liu Y, Dorn GW II. Mitochondrial fusion is essential for organelle function and cardiac homeostasis. Circ Res 2011;109:1327-1331.

34. Chen Y, Dorn GW II. PINK1-phosphorylated mitofusin 2 is a Parkin receptor for culling damaged mitochondria. Science 2013;340:471-475.

35. Lesnefsky EJ, Chen Q, Hoppel CL. Mitochondrial metabolism in aging heart. Circ Res 2016;118:1593-1611.

36. Youle RJ, and, Narendra DP. Mechanisms of mitophagy. Nat Rev Mol Cell Biol 2011; 12:9-13.

37. Eisenberg-Lerner A, Kimchi A. The paradox of autophagy and its implication in cancer etiology and therapy. Apoptosis 2009;14:376-391.

38. Cao DJ, Jiang N, Blagg A, Johnstone JL, Gondalia R, Oh M, Luo X, Yang KC, Shelton JM, Rothermel BA, Gillette TG, Dorn GW, Hill JA. Mechanical unloading activates FoxO3 to trigger Bnip3-dependent cardiomyocyte atrophy. J Am Heart Assoc 2013;2: e000016.

39. Attaix D, Bechet D. FoxO3 controls dangerous proteolytic liaisons. Cell Metab 2007; 6:425-427.

\section{Translational perspective}

The aged heart is more susceptible to stress despite the increased compensatory chaperones/co-chaperones activity. Here, we identified a point mutation in HSPD1 in a human DCM family. Using zebrafish, we demonstrated that functional inactivation of HSPD1 resulted in increased ROS level and mitophagy, thereby resulting in heart failure at a relatively early age. Inhibition of ROS activity by antioxidants decreased cell death and mitophagy. This work identifies the key role of HSPD1 in cardiac muscle protection and suggests the supplementation of antioxidants may improve the cardiac function through the mitochondrial ROS pathway in patients with chronic heart failure. 